\title{
A phase II study of combined chemotherapy with methotrexate, 5-fluorouracil, and low-dose cisplatin (MFP) for histologically diffuse-type advanced and recurrent gastric cancer (KDOG9501)
}

\author{
Norisuke Nakayama, Wasaburo Koizumi, Satoshi Tanabe, Tohru Sasaki, and Katsunori Saigenji \\ Kitasato Digestive Disease and Oncology Group (KDOG) / Department of Gastroenterology, Kitasato University School of Medicine, \\ 1-15-1 Kitasato, Sagamihara, Kanagawa 228-8555, Japan
}

\begin{abstract}
Background. Histologically diffuse-type gastric cancer is well known to have a poor prognosis and is often complicated with abdominal and pleural effusions. We evaluated the efficacy of a low dose of cisplatin combined with methotrexate and 5fluorouracil (MFP therapy) in diffuse-type advanced gastric cancer.

Methods. The study group comprised 47 patients. Patients with pleural effusion or ascites were actively enrolled. Methotrexate $\left(30 \mathrm{mg} / \mathrm{m}^{2}\right.$ per day) plus 5-fluorouracil $\left(600 \mathrm{mg} / \mathrm{m}^{2}\right.$ per day) was administered on days 1 and 8 . Cisplatin $\left(6 \mathrm{mg} / \mathrm{m}^{2}\right.$ per day) was given daily for 14 days after which there was 14 day's rest. Calcium leucovorin (15 mg) was administered after methotrexate.

Results. The overall response rate was $38.3 \%$. Ascites disappeared or decreased in 15 of 23 patients (disappeared in 9). Pleural effusion disappeared or decreased in 6 of 8 patients (disappeared in 4). Of the 21 patients with poor oral intake at study entry, $15(71.4 \%)$ were able to orally ingest food. Hematologic toxicity of grade 3 or higher comprised leukopenia in 10 patients $(21.3 \%)$ and neutropenia in $9(19.1 \%)$. Nonhematologic toxicity included anorexia and nausea/vomiting, in 1 patient each $(2.1 \%)$. The median survival time was 211 days.

Conclusion. MFP therapy is useful for the management of diffuse-type inoperable and recurrent gastric cancer, even in patients with conditions such as pleural effusion, ascites, or lymphangitis carcinomatosa who have a poor prognosis or cannot eat solid food.
\end{abstract}

Key words Histologically diffuse-type gastric cancer · Combination chemotherapy

\section{Introduction}

Biochemical modulation refers to the enhancement of antitumor activity by drug-induced biochemical alter-

Offprint requests to: $\mathrm{N}$. Nakayama

Received: August 5, 2005 / Accepted: February 21, 2006 ations of tumor cell metabolism. Sequential treatment with methotrexate and 5-fluorouracil, a regimen based on the principle of biochemical modulation, was proposed by Bertino et al. [1] in 1977 for the management of colorectal cancer. This regimen was introduced to Japan in 1980 for the treatment of gastric cancer [2] and has been widely used and confirmed to be beneficial. The combination of cisplatin and 5-fluorouracil is, similarly, based on the concept of biochemical modulation. Cisplatin not only acts as an effector, but also augments the antitumor activity of 5-fluorouracil [3,4].

Several clinical trials have evaluated the response of advanced gastric cancer to sequential methotrexate and 5-fluorouracil. Dickinson et al. [5] reported a response rate of $22 \%(5 / 23)$ and a median survival of 11 months. Phase II studies done by Blijham et al. [6] reported a response rate of $18 \%(4 / 22)$ and a median survival of 4 months. A previous Japanese phase II study of sequential methotrexate/5-fluorouracil therapy against advanced gastric cancer demonstrated that lowand intermediate-dose methotrexate/5-fluorouracil regimens achieved response rates of 23\% (13 partial responses [PRs]/56 patients) and $41 \%(15 \mathrm{PRs} / 37$ patients), respectively [7]. Sasaki et al. [8] reported that an intermediate-dose methotrexate/5-fluorouracil regimen achieved a response rate of $17.9 \%$ (10 PRs/56 patients), and Konishi et al. [2] reported a response rate of $23.7 \%$ (9/38). These findings suggest that sequential methotrexate/5-fluorouracil therapy may be effective in advanced gastric cancer.

Combination chemotherapy with cisplatin and 5fluorouracil has also been evaluated in patients with advanced gastric cancer. A phase II trial done by Lacave et al. [9] reported a response rate of $41 \%$ (22/ 53), with a median survival of 10.6 months. A phase III study comparing 5-fluorouracil plus cisplatin (FP) with 5-fluorouracil alone and a combination of 5fluorouracil, doxorubicin, and mitomycin $\mathrm{C}$, done by Kim et al. [10], obtained a response rate of $51 \%$ for 5 - 
fluorouracil plus cisplatin, with a median survival of 8 months. A phase II study done by Rouger et al. [11] reported a response rate of $43 \%(36 / 83)$ and a median survival of 9 months. Ohtsu et al. [12] performed a phase II study and reported a response rate of $43 \%$ (17/ 40 ) and a median survival of 7 months.

Histologically diffuse-type gastric cancer is well known to have a poor prognosis and is often complicated with abdominal and pleural effusions. Few studies support the usefulness of chemotherapy for the treatment of diffuse-type gastric cancer. But Konishi et al. [2] reported that histologically diffuse-type gastric cancer showed a better response than the intestinal type when treated with sequential methotrexate and 5-fluorouracil. Moreover, in-vitro experiments have demonstrated that diffuse-type gastric cancer is sensitive to cisplatin, suggesting antitumor activity in this type [13]. These findings provide evidence that sequential methotrexate and 5-fluorouracil combined with cisplatin may be effective clinically against histologically diffuse-type gastric cancer. We assessed the response of inoperable and recurrent histologically diffuse-type gastric cancer to sequential methotrexate and 5-fluorouracil given concurrently with cisplatin (MFP), serving as an effector. The primary endpoint planned for this study was the tumor response rate. Secondary endpoints were overall survival, response rate in patients with asctes and pleural effusion, and response rate in patients with poor oral intake.

\section{Patients and methods}

\section{Patient eligibility}

The study group comprised patients with inoperable or recurrent gastric cancer treated at our hospital between 1995 and 2001. Eligibility criteria were as follows: (1) all patients had a histopathological diagnosis of diffusetype adenocarcinoma, as confirmed by endoscopic biopsy; (2) unresectable or recurrent disease; (3) no other cancers were identified; (4) the patient was not more than 85 years of age at entry; (5) performance status was 0 to 2 according to the World Health Organization (WHO) criteria; (6) adequate organ function; (7) there was no serious concurrent disease; and (8) informed consent was obtained from all patients prior to entry. Patients with pleural effusion or ascites (including massive ascites) and lymphangitis carcinomatosa were also actively enrolled. Malignant ascites and pleural effusion were confirmed by computed tomography (CT) and cytology. Effusions were not drained prior to or during the course of the study.

\section{Treatment methods}

An intravenous injection of methotrexate $\left(30 \mathrm{mg} / \mathrm{m}^{2}\right)$ was followed $3 \mathrm{~h}$ later by an intravenous infusion of 5 -fluorouracil $\left(600 \mathrm{mg} / \mathrm{m}^{2}\right)$, given over the course of $15 \mathrm{~min}$. Twenty-four $\mathrm{h}$ after the treatment with methotrexate, calcium leucovorin $(15 \mathrm{mg})$ was administered, at 6-h intervals, for a total of six times. To prevent renal dysfunction due to methotrexate, patients received an intravenous infusion of $7 \%$ sodium bicarbonate $(120 \mathrm{ml})$, given at 6 -h intervals, starting $3 \mathrm{~h}$ before the injection of methotrexate. Immediately after the treatment with methotrexate, acetazolamide $(250 \mathrm{mg})$ was given by intravenous injection, to alkalinize the urine. This treatment was given on days 1 and 8. Cisplatin ( $6 \mathrm{mg} / \mathrm{m}^{2}$ per day, 1-h infusion) was administered daily on days 1 to 14 . No chemotherapy was given on days 15 to 28 . This cycle was repeated.

\section{Evaluation of response}

The tumor response was evaluated based on changes in the size of measurable lesions and assessment of evaluable lesions. Measurable lesions and assessment of evaluable lesions were evaluated according to the Japanese Criteria for Evaluating the Efficacy of Chemotherapy and Radiation Therapy in the Treatment of Gastric Cancer [14].

A complete response (CR) was defined as the disappearance of all evidence of cancer for at least 4 weeks, and a PR was defined as less than complete, but more than $50 \%$ reduction of tumor volume for at least 4 weeks, without any evidence of new lesions or progression. No change (NC) was defined as less than a $50 \%$ reduction or less than a $25 \%$ increase without any new lesions. Progressive disease (PD) was defined as a more than $25 \%$ increase in a solitary lesion or the appearance of new lesions. Tumor measurements were performed every 4 weeks, using CT scans, plain chest X-ray films, upper gastrointestinal endoscopy, and ultrasonography. Primary lesions were classified into the following three categories based on X-ray and endoscopic findings: measurable, not measurable but evaluable, and diffuse infiltration. Ascites and pleural effusion were evaluated by CT, as disappeared (no evidence of ascites or effusion for 4 consecutive weeks), decreased (a decrease for 4 consecutive weeks), or no response. The responses of effusions and lymphangitis were not counted in the overall response. Adverse events were evaluated according to World health Organization (WHO) criteria [15].

\section{Statistical considerations}

The required sample size for this study was calculated based on a target response rate of $40 \%$ and a minimum 
response rate of $20 \%$, with an $\alpha$ error of 0.1 and a $\beta$ error of 0.1. But, during the study, because the response rate was high, we enlarged the sample size. Survival was defined as the interval from the day of starting the MFP therapy to death. Survival was estimated according to the Kaplan-Meier method [16].

\section{Results}

\section{Patient characteristics}

Forty-seven patients (29 men and 18 women) were enrolled in this study. Histologically, all tumors were diffuse type. Eight patients had received prior chemotherapy (5-fluorouracil alone, $n=1$; 5-fluorouracil and cisplatin, $n=2$; cisplatin, mitomycin $\mathrm{C}$, and doxifluridine, $n=1$; 5-fluorouracil, mitomycin $\mathrm{C}$, and cisplatin, $n=1$; capecitabine, $n=1$; tegafur-uracil [UFT], $n=1$; and UFT and cisplatin, $n=1$ ). Median age was 62 years (range, 28-84 years). Performance status ranged from 0 to $2(0, n=10 ; 1, n=13 ; 2, n=24)$. Twenty-one patients could not orally ingest food. Ascites was present in 23 patients $(48.9 \%)$ and pleural infusion in 8 $(17.0 \%)$. Lymphangitis carcinomatosa was present in 2 patients $(4.3 \%)$. The demographic characteristics of all patients are shown in Table 1.

\section{Response to chemotherapy and survival}

In total, 141 administrations of MFP therapy were performed in 47 patients. The median number of administrations was 3 (range, 1-7). Among the 47 patients with evaluable lesions, the overall response rate was $38.3 \%$ (18/47; 95\% confidence interval [CI], 24.4\%-52.2\%). Ascites disappeared or decreased in 15 of 23 patients (65.2\%; 95\% CI, 45.8-84.7\%) including complete disappearance in 9. Pleural effusion disappeared or decreased in 6 of 8 patients $(75.0 \%)$ including complete disappearance in 4 (Table 2). Oral ingestion of food became possible in 15 of the 21 patients $(71.4 \%)$ who were previously unable to orally ingest food. The survival curve is shown in Fig. 1. Median survival time was 211 days (95\% CI, 145-277 days) and the 1- and 2-year survival rates were 28.9 and $4.4 \%$, respectively. The overall response rate and median survival time in the 23 patients with ascites were $43.5 \%(10 / 23 ; 95 \%$ CI, $23.2 \%-63.7 \%$ ) and 211 days (95\% CI, 114-308 days), respectively.

\section{Adverse events}

The major treatment-induced hematologic and nonhematologic adverse events are shown in Table 3 . Hematologic toxicity of grade 3 or higher comprised
Table 1. Patient characteristics $(n=47)$

\begin{tabular}{|c|c|c|}
\hline & $n$ & Percentage \\
\hline \multicolumn{3}{|l|}{ Age (years) } \\
\hline Median (range) & $62(28-84)$ & \\
\hline \multicolumn{3}{|l|}{ Sex } \\
\hline Male & 29 & 61.7 \\
\hline Female & 18 & 38.3 \\
\hline \multicolumn{3}{|l|}{ Performance status } \\
\hline 0 & 10 & 21.3 \\
\hline 1 & 13 & 27.7 \\
\hline 2 & 24 & 51.0 \\
\hline \multicolumn{3}{|l|}{ Histology } \\
\hline Diffuse & 47 & 100 \\
\hline Intestinal & 0 & 0 \\
\hline \multicolumn{3}{|l|}{ Prior chemotherapy } \\
\hline None & 39 & 82.9 \\
\hline $5-\mathrm{FU}$ & 1 & 2.1 \\
\hline FP (5-FU, CDDP) & 2 & 4.3 \\
\hline CDDP, MMC, 5'DFUR & 1 & 2.1 \\
\hline FMP (5-FU, MMC, CDDP) & 1 & 2.1 \\
\hline Capecitabine & 1 & 2.1 \\
\hline UFT & 1 & 2.1 \\
\hline $\mathrm{UFT}+\mathrm{CDDP}$ & 1 & 2.1 \\
\hline \multicolumn{3}{|l|}{ Surgical resection } \\
\hline Yes & 4 & 8.5 \\
\hline No & 43 & 91.5 \\
\hline \multicolumn{3}{|l|}{ Oral intake } \\
\hline Good & 26 & 55.3 \\
\hline Poor & 21 & 44.7 \\
\hline \multicolumn{3}{|l|}{ Metastasis } \\
\hline Liver & 9 & 19.1 \\
\hline Lymph nodes & 39 & 82.9 \\
\hline Ovary & 4 & 8.5 \\
\hline Lung & 2 & 4.3 \\
\hline Ascites & 23 & 48.9 \\
\hline Pleural effusion & 8 & 17.0 \\
\hline Lymphangitis carcinomatosa & 2 & 4.3 \\
\hline \multicolumn{3}{|l|}{ No. of treatment cycles } \\
\hline Median (range) & $3(1-7)$ & \\
\hline
\end{tabular}

Performance status according to the grading system of the World Health Organization

leukopenia in 10 patients $(21.3 \%)$ and neutropenia in $9(19.1 \%)$. Nonhematologic toxicity of grade 3 or higher was shown for anorexia and nausea/vomiting in 1 patient each $(2.1 \%)$. Hematologic toxicity was controlled through the use of granulocyte colony-stimulating factor (G-CSF), and no major problems occurred. There were no treatment-related deaths.

\section{Discussion}

Methotrexate is a folic acid antagonist that blocks dihydrofolate reductase (DHFR), an enzyme catalyzing the conversion of dihydrofolate (FH2) to tetrahydrofolate (FH4) [17]. Nucleic acid synthesis is, consequently, blocked. However, monotherapy with methotrexate is of limited effectiveness for gastric can- 
Table 2. Responses

\begin{tabular}{|c|c|c|c|c|c|}
\hline & $\mathrm{CR}$ & PR & $\mathrm{NC}$ & PD & Response rate \\
\hline Overall & 0 & 18 & 20 & 9 & $38.3 \%(18 / 47)$ \\
\hline Primary & 0 & 17 & 26 & 4 & $36.2 \%(17 / 47)$ \\
\hline Liver & 1 & 4 & 2 & 2 & $55.6 \%(5 / 9)$ \\
\hline Lymph nodes & 3 & 14 & 16 & 6 & $43.6 \%(17 / 39)$ \\
\hline Lung & 0 & 1 & 0 & 1 & $50.0 \%(1 / 2)$ \\
\hline \multirow[t]{2}{*}{ Ovary } & 0 & 2 & 2 & 0 & $50.0 \%(2 / 4)$ \\
\hline & Disappeared & & ed & No response & \\
\hline Ascites & 9 & & & 8 & $65.2 \%(15 / 23)$ \\
\hline Pleural effusion & 4 & & & 2 & $75.0 \%(6 / 8)$ \\
\hline Lymphangitis & & & & 1 & $50.0 \%(1 / 2)$ \\
\hline
\end{tabular}

$\mathrm{CR}$, complete response; $\mathrm{PR}$, partial response; $\mathrm{NC}$, no change; $\mathrm{PD}$, progressive disease

Table 3. Toxicity

\begin{tabular}{lrrrrr}
\hline & \multicolumn{5}{c}{ WHO grade } \\
\cline { 2 - 6 } & 1 & 2 & 3 & 4 & 3 or 4 \\
\hline Leukopenia & 4 & 10 & 7 & 3 & $21.3 \%$ \\
Neutropenia & 2 & 9 & 6 & 3 & $19.1 \%$ \\
Anemia & 5 & 3 & & & \\
Thrombocytopenia & 1 & 9 & & & \\
AST & 2 & 1 & & & \\
ALT & 2 & 1 & & & \\
Alkaline phosphatase & 2 & 1 & & & \\
Creatinine & 1 & & & \\
Nausea/Vomiting & 8 & 2 & 1 & & $2.1 \%$ \\
Anorexia & 4 & 2 & 1 & & \\
Diarrhea & 3 & 2 & & & \\
Stomatitis & 3 & & & & \\
Alopecia & 1 & & & \\
Peripheral neuropathy & 1 & & & \\
WHO,World Heath Organy & & & &
\end{tabular}

WHO, World Health Organization; AST, aspartate aminotransferase; ALT, alanine aminotransferase

cer [18]. The mechanism of sequential methotrexate and 5-fluorouracil, a regimen based on the principle of biochemical modulation, has been described by Bertino et al. [1] and Fernandes and Bertino [19]. Pretreatment with the folic acid antagonist methotrexate increases the production of dihydrofolate polyglutamate, which strongly binds to 5-fluoro-2'-deoxyuridine 5 '-monophosphate (5-FdUMP) and thymidylate synthetase (TS), forming a terminal complex that inactivates TS, thereby attenuating DNA synthesis. Cadman et al. $[20,21]$ have reported that methotrexate triggers the blockade of purine synthesis, resulting in the intracellular accumulation of phosphoribosyl pyrophosphate (PRPP). Because PRPP serves as a substrate for the conversion of 5-fluorouracil into 5-fluorouridinemonophosphate (5-FUMP), a reaction catalyzed by orotate phosphoribosyl transferase (OPRT), metho-

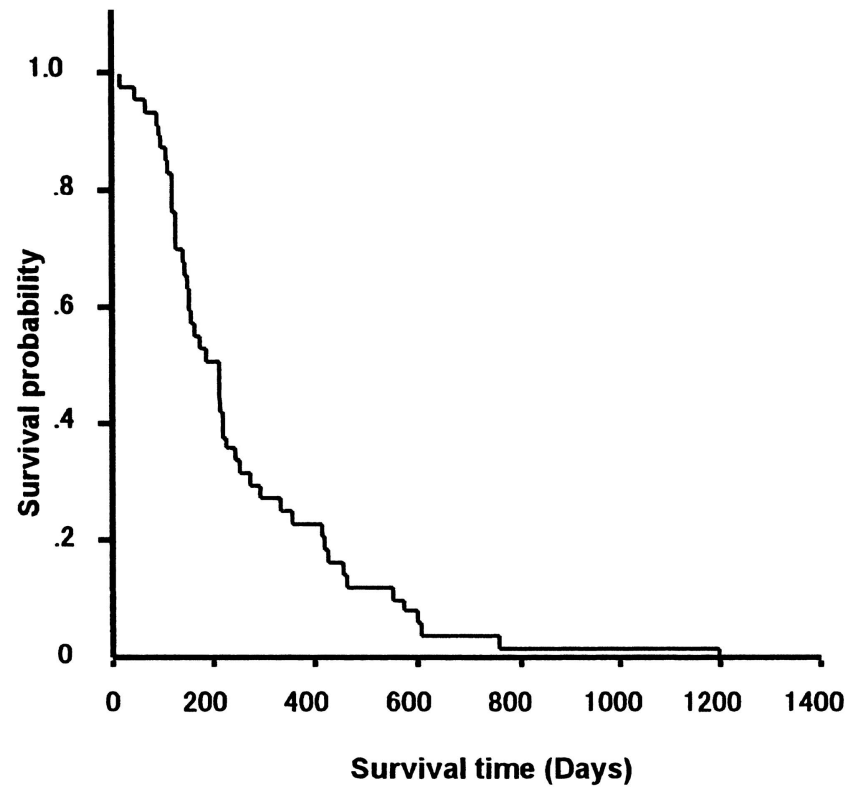

Fig. 1. Overall cumulative survival rate after treatment. The median survival time was 211 days $(95 \%$ confidence interval [CI]; 145-277 days)

trexate pretreatment promotes the conversion of FUMP to 5-fluorouridine 5'-triphosphate (FUTP). Large amounts of 5-FUTP are then incorporated into RNA, enhancing antitumor activity.

Sequential methotrexate and 5-fluorouracil has been shown to be effective against histologically diffuse-type gastric cancer $[2,22,23]$. TS activity is high in diffusetype gastric cancer and low in the highly differentiated type, whereas thymidine kinase (TK) activity is low in diffuse-type gastric cancer and high in the highly differentiated type [23]. Studies examining the relation between TS expression and the response to leucovorin plus 5-fluorouracil have reported that high expression is associated with a low response rate [24]. DNA synthesis 
is suppressed in cells with high TS activity and is disturbed in cells with low TS activity [25]. Available evidence thus suggests that sequential methotrexate and 5-fluorouracil acts by functionally impairing RNA, and is thereby effective against diffuse cancer.

We concomitantly administered a low dose of cisplatin with sequential methotrexate and 5fluorouracil. Cisplatin inhibits DNA synthesis by binding, in a bridge-like fashion, to bases between the double strands of DNA, killing tumor cells. The response rate of gastric cancer to cisplatin monotherapy ranges from $18 \%$ to $33 \%$ [26-30]. In-vitro experimental studies have shown that diffuse-type cancer is sensitive to cisplatin, suggesting antitumor effectiveness in this type [13]. Clinically, Lacave et al. [31] reported that remissions achieved by cisplatin monotherapy in patients with gastric cancer were obtained not only in the intestinal type but also in the diffuse type. Hainsworth et al. [32] recommended cisplatin-based combination chemotherapy for the treatment of poorly differentiated carcinoma and adenocarcinoma of an unknown primary site. The results of these studies suggest that cisplatin is effective for diffuse-type gastric cancer. However, large doses of cisplatin can cause severe toxicity, including gastrointestinal symptoms and renal dysfunction. Moreover, renal toxicity requires the administration of large volumes of infusion solutions. Drewinko et al. [33] showed that the exposure of lymphoma cells to $50 \mu \mathrm{g} / \mathrm{ml}$ of cisplatin for $1 \mathrm{~h}$ or to $5 \mu \mathrm{g} / \mathrm{ml}$ of cisplatin for $8 \mathrm{~h} \mathrm{had} \mathrm{similar} \mathrm{cytocidal}$ activity in vitro. Belliveau et al. [34] reported that continuous infusion of cisplatin for 5 days had a 40 times lower maximal plasma concentration (Cmax) and an approximately two times greater area under the plasmaconcentration-time curve than did an equivalent dose given as a single 30-min infusion. These reports suggest that the administration of cisplatin in small divided doses reduces the risk of toxicity such as renal dysfunction, by lowering the peak plasma concentration of cisplatin, without compromising antitumor effectiveness. In Japan, the dose of cisplatin used in monotherapy for gastric cancer is $70-90 \mathrm{mg} / \mathrm{m}^{2}$ per month. One-day administration of the same dosage requires a large amount of infusion to prevent cisplatin-induced renal injury. This is, however, inappropriate for patients who have peritoneal dissemination and retention of ascites, because such patients may have renal impairment or poor performance status. We therefore fixed the dose of cisplatin at $6 \mathrm{mg} / \mathrm{m}^{2}$ per day for 14 days $\left(84 \mathrm{mg} / \mathrm{m}^{2}\right.$ per month). Because intermediate-dose methotrexate has greater toxicity than low-dose methotrexate, and because MFP is a triple chemotherapy, we adopted lowdose methotrexate and cisplatin, because we wanted to reduce the risk of toxicity.

Peritoneal dissemination is more frequent in patients with undifferentiated carcinoma than in those with dif- ferentiated carcinoma. Konishi et al. [2] reported that histologically diffuse-type gastric cancer showed a better response than the intestinal type to sequential methotrexate/5-fluorouracil treatment. They reported that this sequential therapy was effective even in patients with ascites, with a response rate in patients with ascites of $57 \%(8 / 14)$ [35].

We therefore combined low-dose cisplatin with sequential methotrexate and 5-fluorouracil in patients with histologically diffuse-type gastric cancer, which is often associated with ascites and pleural effusion, conditions in which large infusion volumes are contraindicated.

Our overall response rate with MFP was 38.3\% (18/ 47). The overall response rate in the 23 patients with inoperable or recurrent gastric cancer who had ascites was $43.5 \%$ (10/23), with 15 patients showing a distinct decrease in ascitic fluid (including 9 with complete disappearance of ascites). Yamao et al. [36] reported that sequential methotrexate and 5-fluorouracil distinctly decreased ascites in 13 of 37 patients (35.1\%, including 3 with complete disappearance of ascites) who had inoperable or recurrent gastric cancer with malignant ascites. Tahara et al. [37] demonstrated a clearcut decrease in ascites in 13 of 26 patients with gastric cancer $(50.0 \%$; 5 with complete disappearance of ascites) who received sequential methotrexate and 5fluorouracil. Pleural effusion distinctly decreased in 6 of 8 patients $(75.0 \%)$, including 4 with complete resolution. These results indicate that MFP is effective in patients who have ascites and pleural effusion associated with advanced gastric cancer.

Konishi et al. [38] gave sequential methotrexate and 5-fluorouracil to patients who had gastric cancer with ascites; they reported that the serum methotrexate concentration was maintained at higher levels in the presence of ascites than in the absence of ascites. The concentration of methotrexate in ascitic fluid was higher than that in serum from 6 to $72 \mathrm{~h}$ after treatment. Similar methotrexate levels are expected in patients given MFP. High penetration of methotrexate to ascitic fluid results in delayed excretion, but is considered responsible for the decreases in ascites and pleural effusion, with the attendant improvement in symptoms. We therefore recommend MFP for patients with ascites or pleural effusion.

Of our 21 patients who were unable to eat solid food at study entry, $15(71.4 \%)$ were able to orally ingest food after MFP. This regimen can thus be used in patients who cannot orally ingest food or anticancer agents, and our results suggest that the activities of daily living are improved substantially. To reduce the risk of toxicity, the patients' urine was alkalinized by treatment with sodium bicarbonate or acetazolamide to promote methotrexate excretion. On the days that patients 
received methotrexate, nonsteroidal anti-inflammatory drugs were avoided [39].

Our response rate with MFP was relatively high, but the mean survival time was 211 days (95\% CI, 145-277 days). However, given that all patients had histologically diffuse-type cancer, including many with complications such as ascites, pleural effusion, and lymphangitis, the median survival time was considered acceptable and toxicity tolerable. The median survival time of patients with ascites was also 211 days (95\% CI, 114-308 days). This result was compatible with the results in studies by Yamao et al. [36] and Tahara et al. [37], who reported the median survival times of patients with ascites as 155 days and 244 days after MF tharapy, respectively.

The major adverse events in our study were leucopenia and neutropenia. Hematologic toxicity of grade 3 or higher comprised leukopenia in 10 patients $(21.3 \%)$ and neutropenia in $9(19.1 \%)$. Nonhematologic toxicity of grade 3 or higher constituted anorexia and nausea/ vomiting in 1 patient each $(2.1 \%)$. As reported previously, the hematologic toxicity of MF therapy, of grade 3 or worse leucopenia and neutropenia, ranged from $15 \%$ to $27 \%$. Nonhematologic toxicity of grade 3 or higher anorexia and nausea/vomiting was observed in $5 \%-12 \%$ of patients and $5 \%-11 \%$ of patients $[7,8,36,37]$. These reports suggest that the toxicity of MFP is tolerable.

MFP may be considered an option for therapy in patients with histologically diffuse-type gastric cancer, including those with pleural effusion, and ascites or poor oral intake. Although the results of this pilot study are very promising, controlled clinical trials with other standard regimens are necessary before firm conclusions can be made.

\section{References}

1. Bertino JR, Sawicki WL, Lindquist CA, Gupta VS. Scheduledependent antitumor effects of methotrexate and 5-fluorouracil. Cancer Res 1977;37:327-8.

2. Konishi T, Hiraishi M, Mafune K, Miyama T, Hirata T, Mori K, et al. Therapeutic efficacy and toxicity of sequential methotrexate and 5-fluorouracil in gastric cancer. Anticancer Res 1994;14:12779.

3. Scanlon KJ, Newman EM, Lu Y, Priest DG. Biochemical basis for cisplatin and 5-fluorouracil synergism in human sovarian carcinoma cells. Proc Natl Acad Sci USA 1986;83:89235.

4. Shirasaka T, Shimamoto Y, Ohshimo H, Saito H, Fukushima M. Metabolic basis of the synergistic antitumor activities of 5fluorouracil and cisplatin in rodent tumor models in vivo. Cancer Chemother Pharmacol 1993;32:167-72.

5. Dickinson R, Presgrave P, Levi J, Milliken S, Woods R. Sequential moderate-dose methotrexate and 5-fluorouracil in advanced gastric adenocarcinoma. Cancer Chemother Pharmacol 1989;24: $67-8$.

6. Blijham G, Bleiberg H, Duez N, Buyse M. An EORTC phase II study of sequential methotrexate-fluorouracil in locally advanced or metastatic gastric cancer. The EORTC Gastrointestinal Cancer Cooperative Group. Eur J Cancer 1990;26:63-5.

7. Murakami M, Ota K, Miyazaki T, Niitsu Y, Wakui A, Yokoyama $\mathrm{M}$, et al. Sequential methotrexate - 5-fluorouracil (MTX-5FU) treatment for patients with advanced gastric and colorectal cancer (in Japanese with English abstract). Jpn J Cancer Chemother 1987;14:2482-90.

8. Sasaki T, Ota K, Ibayashi J, Sakata Y, Matsuoka T, Ishikawqa M, et al. Randomized muticenter trial: sequential methotrexate and 5-fluorouracil (MTX-5FU) versus 5-fluorouracil alone in advanced gastric and colorectal cancer (in Japanese with English abstract). Jpn J Cancer Chemother 1989;16:2545-55.

9. Lacave AJ, Baron FJ, Anton LM, Estrada E, De Sande LM, Palacio I, et al. Combination chemotherapy with cisplatin and 5fluorouracil 5-day infusion in the therapy of advanced gastric cancer: A phase II trial. Ann Oncol 1991;2:751-4.

10. Kim NK, Park YS, Heo DS, Suh C, Kim SY, Park KC, et al. A phase III randomized study of 5-fluorouracil and cisplatin versus 5-fluorouracil, doxorubicin, and mitomycin $\mathrm{C}$ versus 5 fluorouracil alone in the treatment of advanced gastric cancer. Cancer 1993;71:3813-18.

11. Rougier P, Ducreux M, Mahjoubi M, Pignon JP, Bellefqih S, Oliveira J, et al. Efficacy of combined 5-fluorouracil and cisplatinum in advanced gastric carcinomas. A phase II trial with prognostic factor analysis. Eur J Cancer 1994;30:1263-9.

12. Ohtsu A, Shimada Y, Yoshida S, Saito H, Seki S, Morise K, et al. Phase II study of protracted infusional 5-fluorouracil combined with cisplatinum for advanced gastric cancer: report from the Japan Clinical Oncology Group (JCOG). Eur J Cancer 1994;30: 2091-3.

13. Maehara Y, Anai H, Kusumoto H, Sugimachi K. Poorly differentiated human gastric carcinoma is more sensitive to antitumor drugs than is well-differentiated carcinoma. Eur J Surg Oncol 1987;13:203-6.

14. Japanese Research Society for Gastric Cancer. Japanese classification of gastric carcinoma. First English edition. Tokyo: Kanehara; 1995.

15. World Health Organization. WHO handbook for reporting results of cancer treatment. Geneva: WHO offset/Publication No. 48; 1979.

16. Kaplan EL, Meier P. Nonparametric estimation from incomplete observations. J Am Stat Assoc 1958;53:457-81.

17. Jolivet J, Cowan KH, Curt GA, Clendeninn NJ, Chabner BA. The pharmacology and clinical use of methotrexate. N Engl J Med 1983;309:1094-104.

18. Bruckner HW, Lokich JJ, Stablein DM. Studies of Baker's antifol, methotrexate, and razoxane in advanced gastric cancer. A Gastrointestinal Tumor Study Group Report. Cancer Treat Rep 1982; 66:1713-17.

19. Fernandes DJ, Bertino JR. 5-Fluorouracil-methotrexate synergy: enhancement of 5-fluorodeoxyridylate binding to thymidylate synthase by dihydropteroylpolyglutamates. Proc Natl Acad Sci USA 1980;77:5663-7.

20. Cadman E, Heimer R, Davis L. Enhanced 5-fluorouracil nucleotide formation after methotrexate administration: explanation for drug synergism. Science 1979;205:1135-7.

21. Cadman E, Heimer R, Benz C. The influence of methotrexate pretreatment on 5-fluorouracil metabolism in L1210 cells. J Biol Chem 1981;256:1695-704.

22. Hironaka SI, Boku N, Ohtsu A, Nagashima F, Sano Y, Muto M, et al. Sequential methotrexate and 5-fluorouracil therapy for gastric cancer patients with bone metastasis. Gastric Cancer 2000; 3:19-23.

23. Konishi T, Miyama T, Sakamoto S, Hirata T, Mafune K, Hiraishi $\mathrm{M}$, et al. Activities of thymidylate synthetase and thymidine kinase in gastric cancer. Surg Oncol 1992;1:215-21.

24. Johnston PG, Lenz HJ, Leichman CG, Danenberg KD, Allegra CJ, Danenberg PV, et al. Thymidylate synthase gene and protein expression correlate and associated with response to 5- 
fluorouracil in human colorectal and gastric tumors. Cancer Res 1995;55:1407-12.

25. Johnston PG, Fisher ER, Rockette HE, Fisher B, Wolmark N, Drake JC, et al. The role of thymidylate synthase expression in prognosis and outcome of adjuvant chemotherapy in patients with rectal cancer. J Clin Oncol 1994;12:2640-7.

26. Beer M, Cocconi G, Ceci G, Varini M, Cavalli F. A phase II study of cisplatin in advanced gastric cancer. Eur J Cancer Clin Oncol 1983;19:717-20.

27. Lacave AJ, Izarzugaza I, Anton Aparicio LM, Valle Pereda M, Gracia Marco JM, Buesa JM. Phase II clinical trial of cisdichlorodiammineplatinum in gastric cancer. Am J Clin Oncol 1983;6:35-8.

28. Leichman L, McDonald B, Dindogru A, Samson M, Vaitkevicius VK. Cisplatin. An active drug in the treatment of disseminated gastric cancer. Cancer 1984;53:18-22.

29. Vogl SE, Camacho FJ, Engstrom PF, Bennett JM. Phase II trial of cisplatin in advanced gastric cancer. Cancer Treat Rep 1984;68: 1497-8.

30. Aabo K, Pedersen H, Rorth M. Cisplatin in the treatment of advanced gastric carcinoma: a phase II study. Cancer Treat Rep 1985;69:449-50.

31. Lacave AJ, Wils J, Diaz-Rubio E, Clavel M, Planting A, Bleiberg $\mathrm{H}$, et al. cis-Platinum as second-line chemotherapy in advanced gastric adenocarcinoma. A phase II study of the EORTC Gastrointestinal Tract Cancer Cooperative Group. Eur J Cancer Clin Oncol 1985;21:1321-4.

32. Hainsworth JD, Johnson DH, Greco FA. Cisplatin-based combination chemotherapy in the treatment of poorly differentiated carcinoma and poorly differentiated adenocarcinoma of unknown primary site: results of a 12-year experience. J Clin Oncol 1992;10: 912-22.

33. Drewinko B, Brown BW, Gottlieb JA. The effect of cisdiamminedichloroplatinum (II) on cultured human lymphoma cells and its therapeutic implications. Cancer Res 1973;33:3091-5.

34. Belliveau JF, Posner MR, Ferrari L, Crabtree GW, Cummings FJ, Wiemann MC, et al. Cisplatin administered as a continuous 5-day infusion: plasma platinum levels and urine platinum excretion. Cancer Treat Rep 1986;70:1215-17.

35. Konishi T, Hirata T, Miyama T, Hiraishi M, Mafune K, Yohida J, et al. Mechanism of synergism and clinical results of sequential methotrexate and 5-fluorouracil in the treatment of gastric cancer (in Japanese with English abstract). Jpn J Cancer Chemother 1992;19:946-53.

36. Yamao T, Shimada Y, Shirao K, Ohtsu A, Ikeda N, Hyodo I, et al. A phase II study of sequential methotrexate and 5-fluorouracil for advanced gastric cancer with malignant ascites: a report from the Gastrointestinal Oncology Study Group of the Japan Clinical Oncology Group, JCOG 9603 Trial. Jpn J Clin Oncol 2004;34: 316-22.

37. Tahara M, Ohtsu A, Boku N, Nagashima F, Muto M, Sano Y, et al. Sequential methotrexate and 5-fluorouracil therapy for gastric cancer patients with peritoneal dissemination: a retrospective study. Gastric Cancer 2001;4:212-8.

38. Konishi T, Noie T, Yoshida J, Mafune K, Makuuchi M. Treatment of peritoneal dissemination of gastric cancer with sequential methotrexate and 5-fluorouracil. Gastric Cancer 1999;2:52-6.

39. Thyss A, Milano G, Kubar J, Namer M, Schneider M. Clinical and pharmacokinetic evidence of a life-threatening interaction between methotrexate and ketoprofen. Lancet 1986;I:256-8. 